\title{
ON DEFINING LONG-RANGE DEPENDENCE
}

\author{
C. C. HEYDE,* Australian National University and Columbia University \\ Y. YANG, ${ }^{* *}$ Columbia University
}

\begin{abstract}
Long-range dependence has usually been defined in terms of covariance properties relevant only to second-order stationary processes. Here we provide new definitions, almost equivalent to the original ones in that domain of applicability, which are useful for processes which may not be second-order stationary, or indeed have infinite variances. The ready applicability of this formulation for categorizing the behaviour for various infinite variance models is shown.

LONG-RANGE DEPENDENCE; PERSISTENCE; FRACTAL MODELS; ALLEN VARIANCE; INFINITE VARIANCE MODELS; FRACTIONAL STABLE NOISE

AMS 1991 SUBJECT CLASSIFICATION: PRIMARY 60G10; 60G18

SECONDARY 62M10
\end{abstract}

\section{Introduction}

The usual definition of long-range dependence is in terms of a second-order stationary process. Let $\left\{X_{t}, t=1,2, \cdots\right\}$ be a zero mean second-order stationary process with covariances

$$
\gamma_{k}=\operatorname{cov}\left(X_{t}, X_{t+k}\right)
$$

and spectral density

$$
f(\omega)=\frac{1}{2 \pi} \sum_{k=-\infty}^{\infty} \gamma_{k} \mathrm{e}^{-\mathrm{i} k \omega}, \quad-\pi<\omega<\pi .
$$

Then, long-range dependence holds if the spectral density is infinite at frequency $\omega=0$, while short-range dependence holds if $f(0)$ is finite. We shall write $\operatorname{LRD}(\mathrm{SD})$ and $\mathrm{SRD}(\mathrm{SD})$ for these definitions, the SD referring to spectral density.

The LRD(SD) definition is inflexible in providing for extensions that allow for departure from stationarity and, indeed, infinite variances. Long-range dependence is a phenomenon which is widely observed in nature and it is characterized by sample paths displaying apparent trends and cycles, although these are ephemeral and are eventually replaced by equally convincing looking ones of quite different apparent direction and

Received 5 November 1996; revision received 11 March 1997.

* Postal address: Stochastic Analysis Group, School of Mathematical Sciences, The Australian National University, Canberra, ACT 0200, Australia and Department of Statistics, Columbia University, New York, NY 10027, USA.

** Postal address: Department of Statistics, Columbia University, New York, NY 10027, USA. 
period. Models in which variances may be infinite are needed for the analysis of a variety of such phenomena (e.g. in finance [5], geology [6], [7], [8] and hydrology [11]). For example, in [11] (e.g. Table 1 therein) the one-dimensional distributions required are of type $\boldsymbol{P}(X>x) \sim C x^{-\beta}$ for large values of $x$, where $\beta$ ranges from 1.10 to 3.22 , while the self-similarity parameter of the process is close to $2 / 3$. In [6], [7], [8], the permeability and porosity of sedimentary rock formations are analyzed and strong evidence is provided for fractional Lévy stable models with index $\alpha, 1<\alpha<2$, and self-similarity parameter $H<1 / \alpha$.

Now consider the block means process $\left\{X_{t}^{(m)}\right\}$ given by

$$
X_{t}^{(m)}=\left(X_{t m-m+1}+\cdots+X_{t m}\right) / m
$$

and the Allen variance

$$
V_{m}=\operatorname{var}\left(X_{t}^{(m)}\right) \text {. }
$$

Then, as has been noted by various authors (e.g. [2]), a nearly equivalent formulation of the dichotomy between SRD(SD) and LRD(SD) is given by $m V_{m} \rightarrow$ finite constant as $m \rightarrow \infty$ (SRD(AV)) and $m V_{m} \rightarrow \infty$ as $m \rightarrow \infty$ (LRD(AV)). Here (AV) refers to definitions with respect to the Allen variance. Indeed, we see that

$$
m V_{m}=\sum_{|j| \leqq m}\left(1-\frac{|j|}{m}\right) \gamma_{j},
$$

so that, using the Kronecker lemma,

$$
m V_{m} \rightarrow \sum_{j=-\infty}^{\infty} \gamma_{j}=\gamma_{0}+2 \sum_{j=1}^{\infty} \gamma_{j}
$$

as $m \rightarrow \infty$ if the right-hand side is finite. Also, if $\Sigma_{|j| \leqq m} \gamma_{j}$ tends to $\pm \infty$ as $m \rightarrow \infty$ then so does $m V_{m}$. However, it is not always assured that $m V_{m} \rightarrow \sigma^{2}$, say, $0<\sigma^{2}<\infty$, will imply that $f(0)$ is finite.

\section{Extensions}

It turns out that the $\mathrm{LRD}(\mathrm{AV})$ definition is readily amenable to extension whereas that for the spectral density lacks similar flexibility. To deal with the possibility of nonstationarity we define a new block means process

$$
Y_{t}^{(m)}=\frac{\sum_{t m-m+1}^{t m} X_{j}}{\sum_{t m-m+1}^{t m} \boldsymbol{E} X_{j}^{2}},
$$

for zero mean variables, and we shall say that the process is LRD(AV) if

$$
\left(\sum_{j=t m-m+1}^{t m} \boldsymbol{E} X_{j}^{2}\right) \operatorname{var} Y_{t}^{(m)} \rightarrow \infty
$$

as $m \rightarrow \infty$. This conveniently allows for departure from stationarity, certainly including the periodic effects associated with seasonality, and it reduces to the original LRD(AV) definition in the stationary case. 
An example of a non-stationary process which is $\mathrm{LRD}(\mathrm{AV})$ is the random walk $\left\{X_{j}=\sum_{k=1}^{j} Z_{k}\right\}$, the increments $\left\{Z_{k}\right\}$ being independent and identically distributed with zero mean and finite variance. This seems an entirely appropriate interpretation as the random walk is well known to produce long leads and rare sign changes despite being 'fair'.

To deal with the situation in which variances may be infinite we suggest replacing the LRD(AV) definition by an empirical version. Suppose that the $X_{j}$ are centered to have zero mean when the mean is finite. We shall say that the process is LRD(SAV), SAV denoting sample Allen variance, if

$$
\frac{\left(\sum_{j=t m-m+1}^{t m} X_{j}\right)^{2}}{\sum_{j=t m-m+1}^{t m} X_{j}^{2}} \stackrel{\mathrm{p}}{\rightarrow} \infty
$$

as $m \rightarrow \infty$. The particular value of this criterion is that it provides a ready basis for statistical testing without a priori knowledge about the variances.

It should be noted that if LRD(SAV) holds for $X_{t}$ stationary with finite variance then

$$
\frac{\left(\sum_{j=t m-m+1}^{t m} X_{j}\right)^{2}}{\sum_{j=t m-m+1}^{t m} X_{j}^{2}} \stackrel{\mathrm{p}}{\rightarrow} \infty \Leftrightarrow m\left(X_{t}^{(m)}\right)^{2} \stackrel{\mathrm{p}}{\rightarrow} \infty \Rightarrow m V_{m} \rightarrow \infty
$$

so that $\mathrm{LRD}(\mathrm{AV})$ holds. Also, $\mathrm{LRD}(\mathrm{SAV})$ holds for the random walk example described above, as can be seen by applying Donsker's invariance principle for the central limit theorem (e.g. [1], pp. 137, 142) separately to numerator and denominator in the definition.

To underscore the value of the LRD(SAV) definition for dealing with infinite variance problems we illustrate by considering the collection of fractional stable noise models discussed by Samorodnitsky and Taqqu [10], Section 7.10. Properties such as covariation ([10], Section 2.7) and codifference ([10], Section 2.10), which are surrogates for the covariance when variances are not finite, do not provide a satisfactory basis for an extension of the standard definition of LRD, although an asymptotic analysis of the codifference for linear fractional stable noise is provided in [10]. In Section 7.10 the authors resort to analogy with the finite variance case to call the process LRD if $H>1 / \alpha, 0<\alpha<2$. They note that the codifference does not even tend to zero as the lag increases for the other models described, namely increments of the real harmonizable stable noise process and the sub-Gaussian fractional stable noise. For fractional stable Lévy noise, Painter [7] refers to the cases $H>1 / \alpha, H<1 / \alpha$ as persistent and anti-persistent (descriptions typically associated with LRD and SRD respectively), again by analogy with the fractional Brownian case.

The models discussed in [10], which essentially form the natural paradigm models for long-range dependence in the infinite variance case are as follows.

(a) The linear fractional stable noise process has increments

$$
\begin{aligned}
X_{j}=\int_{-\infty}^{\infty}\left(a\left[(j+1-x)_{+}^{H-1 / \alpha}-(j-x)_{+}^{H-1 / \alpha}\right]\right. \\
\left.\quad+b\left[(j+1-x)_{-}^{H-1 / \alpha}-(j-x)_{-}^{H-1 / \alpha}\right]\right) M(d x),
\end{aligned}
$$


where $a, b$ are real constants, $|a|+|b|>0,0<\alpha<2,0<H<1, H \neq 1 / \alpha$ and $M$ is an $\alpha$-stable random measure with Lebesgue control measure. Here $a_{+}=\max (0, a)$, $a_{-}=-\min (0, a)$.

(b) The real harmonizable fractional stable noise process has increments

$$
X_{j}=\operatorname{Re} \int_{-\infty}^{\infty} \mathrm{e}^{\mathrm{i} \alpha j} \frac{\mathrm{e}^{\mathrm{i} x}-1}{\mathrm{i} x}|x|^{-H+1-1 / \alpha} \tilde{M}(d x),
$$

where $0<\alpha \leqq 2,0<H<1$ and where $\tilde{M}$ is a complete isotropic symmetric $\alpha$-stable random measure with Lebesgue control measure.

(c) The sub-Gaussian fractional stable noise process has increments

$$
X_{j}=A^{1 / 2}\left(B_{H}(j+1)-B_{H}(j)\right),
$$

where $B_{H}$ is fractional Brownian motion of index $H, 0<H<1$, and $A$ is an independent $\alpha / 2$ stable random variable with Laplace transform $\boldsymbol{E} \mathrm{e}^{-t A}=\mathrm{e}^{-t^{\alpha / 2}}, t>0$. We shall discuss the use of the LRD(SAV) criterion on these models.

In each case it is easily checked that we have self-similarity with parameter $H$, so that

$$
\sum_{t m-m+1}^{t m} X_{j} \stackrel{\mathrm{d}}{=} m^{H} X_{1}
$$

We shall henceforth confine attention to processes $X_{j}$ which have this self-similarity property.

Our basic result on LRD is given in the following theorem.

Theorem 1. Let $X_{j}$ be a stationary process for which block sums are self-similar with parameter $H$ (i.e. satisfy (1)) and suppose that $\boldsymbol{E}\left|X_{1}\right|^{p}<\infty$ for some $0<p<2$. Then $L R D(S A V)$ holds if $H>1 / p$. In particular, if $\boldsymbol{P}\left(\left|X_{1}\right|>x\right) \sim C x^{-\alpha}$ as $x \rightarrow \infty, 0<\alpha<2$, then $L R D(S A V)$ holds if $H>1 / \alpha$.

The result of this theorem follows from the use of the relationship (1) to deal with the numerator in the LRD(SAV) criterion and the stability theorem $\mathrm{E}$ of Loève [4], p. 387 , to deal with the denominator. The stability theorem gives $n^{-2 / p} \sum_{j=1}^{n} X_{j}^{2} \stackrel{\text { a.s. }}{\rightarrow} 0$ as $n \rightarrow \infty$. The particular case is established by noting that $\boldsymbol{E}\left|X_{1}\right|^{p}<\infty$ for all $p<\alpha$.

To explore the LRD/SRD relationship further, we need to examine $L_{p}$ variants of the SAV criterion. Write

$$
F_{m}(p)=\frac{\left|\Sigma_{t m-m+1}^{t m} X_{j}\right|^{p}}{\sum_{t m-m+1}^{t m}\left|X_{j}\right|^{p}}, \quad 0<p \leqq 2 .
$$

Suppose that $X_{j}$ is stationary, ergodic, satisfies the self-similarity condition (1) and $\boldsymbol{E}\left|X_{1}\right|^{p}<\infty$ for $0<p<\alpha<2$. Then, we have that $F_{m}(p) \stackrel{\text { a.s. }}{\rightarrow} \infty$ as $m \rightarrow \infty$ if $p H>1, p<\alpha$, i.e. $1 / H<p<\alpha$. This follows from the use of self-similarity on the numerator and the ergodic theorem on the denominator of $F_{m}(p)$. The result corresponds to the case of long-range dependence as identified by the LRD(SAV) criterion, namely as holding for $H<1 / \alpha$. 
On the other hand, if $\boldsymbol{E}\left|X_{1}\right|^{p}=\infty$ and if $\alpha<p<2, p H<1$, so that $\alpha<p<\min (2,1 / H)$, we have $F_{m}(p) \stackrel{\text { a.s. }}{\rightarrow} 0$ as $m \rightarrow \infty$. To obtain this result we again use self-similarity on the numerator of $F_{m}(p)$ and, for dealing with the denominator we note that, using the ergodic theorem with $I(\cdot)$ for the indicator function,

$$
n^{-1} \sum_{j=1}^{n}\left|X_{j}\right|^{p} \geqq n^{-1} \sum_{j=1}^{n}\left|X_{j}\right|^{p} I\left(\left|X_{j}\right| \leqq k\right) \stackrel{\text { a.s. }}{\rightarrow} E\left|X_{j}\right|^{p} I\left(\left|X_{j}\right| \leqq k\right) \rightarrow \infty
$$

as $k \rightarrow \infty$. The result here corresponds to short-range dependence.

Note that $\alpha<\min (2,1 / H)$ implies $H<1 / \alpha$ (since for $H \geqq 1 / 2, \alpha<\min (2,1 / H)=1 / H$, i.e. $H<1 / \alpha$, while for $H<1 / 2, \alpha<\min (2,1 / H)=2$ and hence $1 / \alpha>1 / 2>H)$. This clarifies the SRD $(H<1 / \alpha)$ and LRD $(H>1 / \alpha)$ dichotomy. We have chosen, however, not to make the $F_{m}(p), p<2$, the basis of a formal definition of LRD/SRD in order to avoid the uncertainty associated with $p$ values which would generally be unknown in practice.

It should be observed that, for the sub-Gaussian fractional stable noise process mentioned above, the dichotomy is $H>1 / 2$ (LRD) and $H<1 / 2$ (SRD). The model here is not ergodic. A single history of the process appears to be from a fractional Brownian motion process and therefore to reflect, as this does, LRD or SRD according to $H>1 / 2$ or $H<1 / 2$. This can easily be seen via the AV or SAV criteria for LRD/SRD. Note that $H>1 / \alpha$ ensures $H>1 / 2$ and the subtlety of ergodic or non-ergodic behaviour does not enter into the LRD consideration of Theorem 1.

Although the formal definition of $\operatorname{LRD}(\mathrm{SAV})$ can be used as a practical criterion for classifying behaviour, it must be used with care as it is based on the assumption of zero means, when these are finite. As an exercise we have investigated the time series of daily returns for the S\&P 500 market index for the period 2 July 1962 to 31 December 1991, the data set having been supplied by a referee. Daily, weekly and monthly series were examined and there is no evidence of LRD. This assessment contrasts with that of Peters [9], p. 113, who used $R / S$ analysis on the returns from the $S \& P 500$ index for the period January 1950 to July 1988 and concluded that the value of the Hurst index $\mathrm{H}$ rises steadily from 0.59 for one-day increments to 0.78 for 30 -day increments. $R / S$ analysis is, however, well-known to be highly sensitive to departures from stationarity (e.g. Heyde and Dai [3]) of the kind that might be expected for financial series.

\section{Acknowledgements}

Helpful comments from a referee and from Huang Dawei are gratefully acknowledged.

\section{References}

[1] Billingsley, P. (1968) Convergence of Probability Measures. Wiley, New York.

[2] Cox, D. R. (1984) Long-range dependence: a review. In Statistics: an Appraisal. ed. H. A. David and H. T. David. Iowa State University Press, Ames. pp. 55-74.

[3] Heyde, C. C. AND DAI, W. (1996) On the robustness to small trends of estimation based on the smoothed periodogram. J. Time Series Anal. 17, 141-150.

[4] LoÈve, M. (1963) Probability Theory. 3rd edn. Van Nostrand, Princeton, NJ.

[5] Mittnik, S. And Rachev, S. T. (1997) Modeling Financial Assets with Alternative Stable Models. Wiley, New York. 
[6] Painter, S. (1995) Random fractal models of heterogeneity: the Lévy-stable approach. Math. Geol. 27, 813-830.

[7] PAINTER, S. (1996) Existence of non-Gaussian scaling behaviour in heterogeneous sedimentary formations. Water Resources Res. 32, 1183-1195.

[8] PAINTER, S. (1996) Stochastic interpolation of aquifer properties using fractional Lévy motion. Water Resouces Res. 32, 1323-1332.

[9] Peters, E. E. (1991) Chaos and Order in the Capital Markets. Wiley, New York.

[10] Samorodnitsky, G. and Taqqu, M. S. (1994) Stable Non-Gaussian Random Processes. Stochastic Models with Infinite Variance. Chapman and Hall, New York.

[11] Turcotte, D. L. (1994) Fractal theory and the estimation of extreme floods. J. Res. Nat. Inst. Stand. Tech. 99, 377-389. 